\title{
Therapeutic effects of vinegar: a review
}

\begin{abstract}
Vinegar is a natural product derived from a process of fermentation. Carbohydrates-rich foods are excellent sources of substrate to produce vinegar. Vinegar is mainly used as an ingredient in food preparation due to its taste and aroma. It is one of the most famous folk medicines used to fight infections. Several studies have showed vinegar has a potential to ameliorate obesity, diabetes, cardiovascular disorders, cancer and microbial infections. Daily intake of a drink containing $15 \mathrm{~mL}$ vinegar (750 mg of acetic acid) was reported to improve lifestyle-related diseases, such as hypertension, hyperlipidemia, and obesity. The presence of acetic acid and other components in vinegar could be responsible for its therapeutic effect. This paper reviews recent studies on therapeutic values of vinegar derived from different food sources. Possible mechanisms of therapeutic action of vinegar are also discussed.
\end{abstract}

Keyword: Vinegar; Therapeutic 